\title{
Packaging Methods of Fiber-Bragg Grating Sensors in Civil Structure Applications
}

\author{
Yung Bin Lin, Kuo Chun Chang, Jenn Chuan Chern, and Lon A. Wang
}

\begin{abstract}
Fiber-Bragg grating (FBG) sensors made on bare fibers are easily damaged when handled improperly during and after fabrication. As a protection from such damage, a novel technique for protecting and packaging FBG sensors has been developed and is presented in this paper. To characterize the strain transmission efficiency of the packaged FBG sensors, an analytical finite-element method is used, and the results are compared with the experiments. It is observed that the thickness and Young's moduli of glues have little influence on the strain transmission, especially when the thickness of the glue is less than the diameter of an optical fiber. However, recoating and steel-tube packaging will markedly affect the strain transmission rate. The strain transmission rates decrease with the increase in thickness of the packaging material. Also, the aging problem of the polymide or acrylate coating and epoxy glue must be considered, since the service life of most structures is usually designed for more than 50 years. The metallic recoated FBG sensor developed in this research uses different approaches, such as low-temperature solder welding, which shows no aging problem, to install the sensors in the structures. Based on the simulated and experimental results, the nickel recoating method is shown to have good strain transmission efficiency compared with other packaging methods.
\end{abstract}

Index Terms-Fiber-Bragg grating (FBG), finite-element model (FEM), packaging, strain.

\section{INTRODUCTION}

$\mathbf{F}$ IBER-grating sensors have attracted a considerable amount of interest in the last ten years for use in optical-fiber sensing applications, such as distributed or quasidistributed measurements of strain, temperature, pressure, acceleration [1]-[9], etc. Compared to other optical-fiber sensors, the fiber grating based sensors have a number of distinct advantages [1], [2] such as wavelength detection, linear response to strain, mass production, ease of multiplexing, remote sensing, and absolute measurement. Fiber-Bragg gratings (FBGs) are easily fabricated through a side exposure technique in two typical configurations, which consist of exposing a small portion of the optical fiber to two interfering beams of UV light or to one UV beam through a phase mask. Either method will create a

Manuscript received September 12, 2002; revised August 5, 2004. This work was supported in part by the Ministry of Transportation and Communications, Taiwan, R.O.C., under Project MOTC-STAO-90-016, and in part by the Ministry of Education Program under Grant 89E-FA06-2-4-7. The associate editor coordinating the review of this paper and approving it for publication was Dr. Subhas Mukhopadhyay.

Y. B. Lin, K. C. Chang, and J. C. Chern are with the Department of Civil Engineering, National Taiwan University, Taipei, Taiwan 10617, R.O.C. (e-mail: yblin@ncree.gov.tw; ciekuo@ntu.edu.tw; jcchern@ntu.edu.tw).

L. A. Wang is with the Department of Electrical Engineering and the Graduate Institute of Electro-Optical Engineering, National Taiwan University, Taipei, Taiwan 10617, R.O.C. (e-mail: lon@ntu.edu.tw).

Digital Object Identifier 10.1109/JSEN.2005.844539 periodic refractive index modulation in the fiber core. Owing to the Bragg resonance condition, only several discrete optical wavelengths will be reflected if incident with a broadband light. The Bragg wavelength of this resonance condition [3], [4] in an FBG can be expressed as

$$
\lambda_{B}=2 n_{\text {eff }} \Lambda .
$$

where $\mathrm{n}_{\text {eff }}$ denotes the effective index of refraction of the fiber core and $\Lambda$ represents the periodicity of the index modulation. As can be seen by (1), any change in the periodicity or in the effective index will change the Bragg wavelength. Consequently, any temperature or strain-inducedeffects on the FBG can be determined by the corresponding shift in the Bragg wavelength.

The strain is determined by measuring the shift of Bragg wavelength $\Delta \lambda$ which is directly related to the axial strain in an optical fiber. From previous experimental studies [5]-[11], it has been demonstrated that the shift of Bragg wavelength has a linear relationship with the applied strain in the axial direction. These FBG sensors have been used in a number of applications to monitor the integrity of aircraft, bridges, buildings, and civil structures. However, an FBG sensor made on a bare fiber is easily damaged when handled improperly during and after fabrication. To prevent such damage, recoating the bare fiber, or providing some protective packaging, is generally desirable [10]-[21]. It must be noted that glue is normally used to mount the FBG sensor onto the host material to measure the strain [10], [12]-[21]. As a result, the aging problem of the polymide or acrylate coating and that of the epoxy glue should be considered, since the service life of most structures is usually designed for more than fifty years. The metallic recoated FBG sensor developed in this study uses different approaches, such as low-temperature solder welding, which shows no aging problem, for installing the sensors in the structures. Moreover, embedding an FBG sensor generally involves a highly alkaline concrete environment ( $\mathrm{pH} 12)$ and requires careful packaging to prevent corrosive damage to the sensor. In addition, the strain transmission needs to be evaluated for different adhesive interfaces between an FBG sensor and its host material.

In this paper, a finite-element model (FEM) with plane stress element simulation is employed to evaluate the adhesive interface effects. An FBG sensor is assumed to be attached to a tension member. The strain transmission efficiency and the corresponding characteristics associated with the thickness and the Young's moduli of the glues are examined. To protect the FBG sensor from damage, novel packaging methods, involving nickel recoating, quartz glue, and steel tubing with 2-mm wall thickness, are developed. The packaged FBG sensors are then surface mounted for measuring the strain of the structure. 


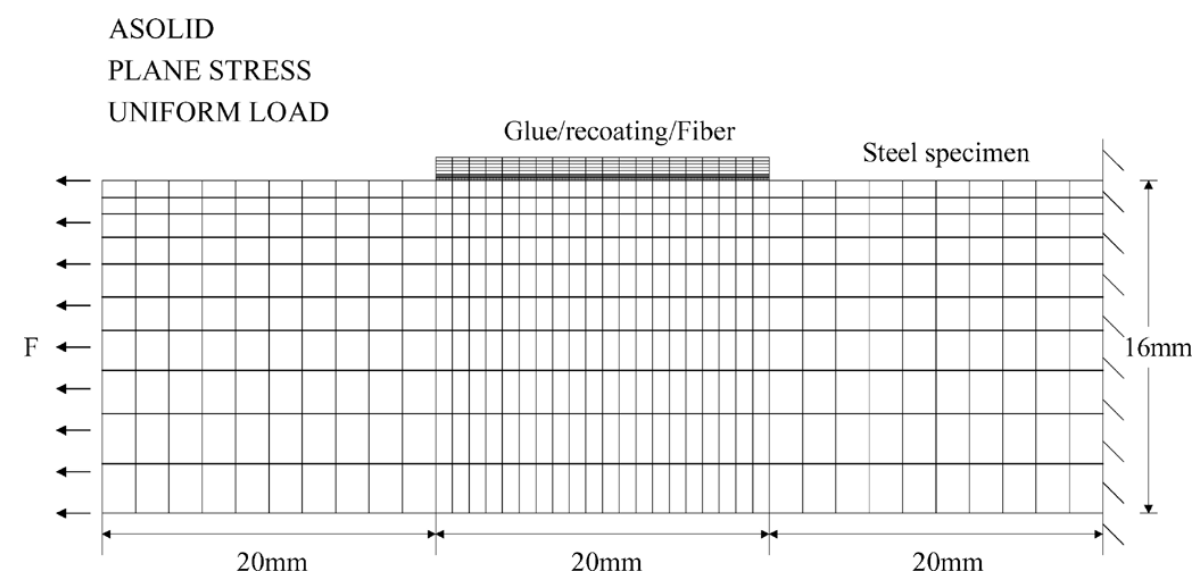

Fig. 1. FBG sensors by FEM model.

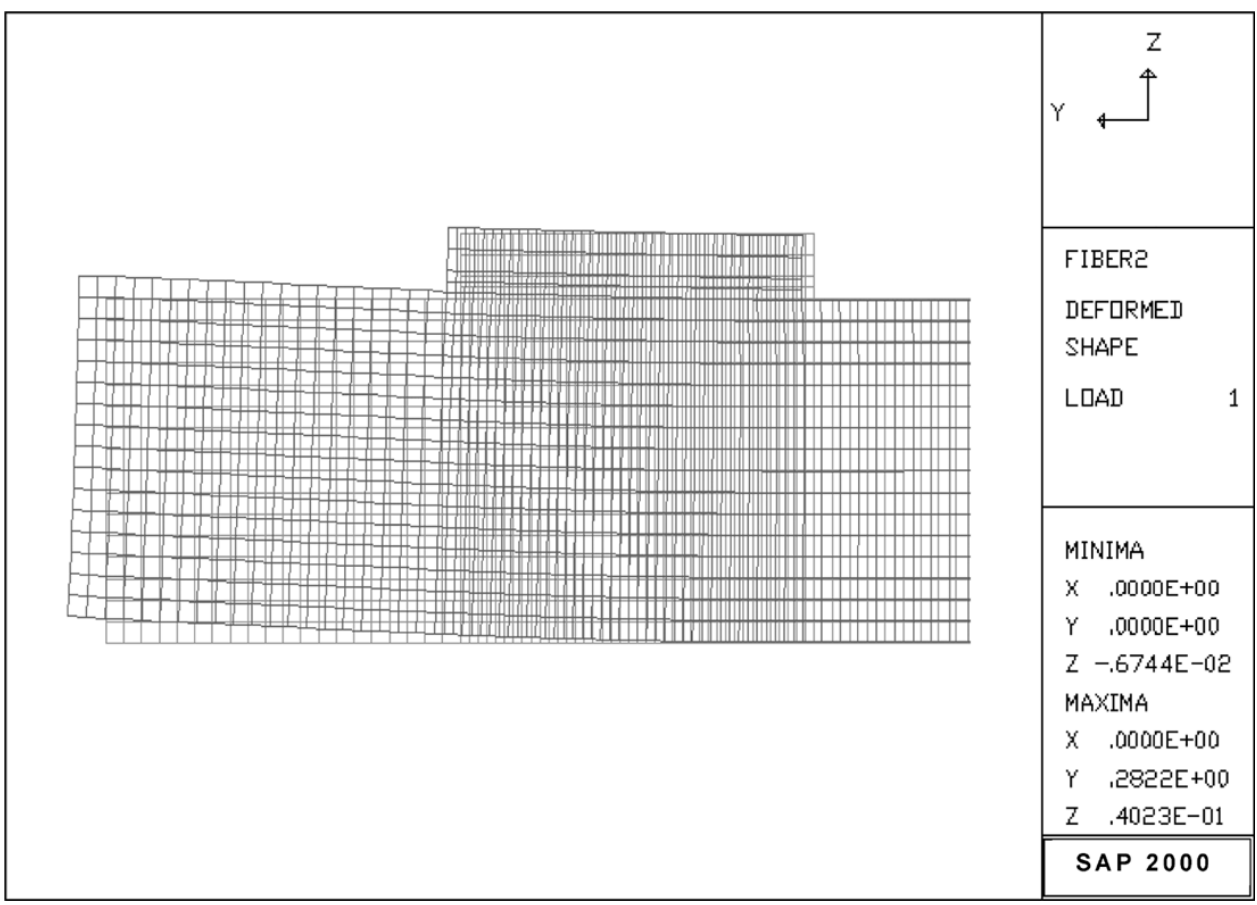

Fig. 2. FEM analysis of the FBG sensors under deformation.

\section{FEM SiMULATION}

To study the effect of different bonding materials for FBG sensors under strain, with and without packaging, and to understand the corresponding strain transmission between the sensor and its host matrix, a FEM using SAP2000 [22] is utilized. The analytical simulation is based on two-dimensional models with plane stress condition. The strain transmission rate of the adhesive interface is investigated for different glues with various thicknesses and Young's moduli. The mesh density of the FEM model is increased from both sides to the interface at a typical geometric ratio in those regions where the strain distribution changes rapidly. The rectangular element mesh, measuring $60 \times 16 \mathrm{~mm}$, is shown in Fig. 1. The FBG sensor, having a length of $20 \mathrm{~mm}$, is attached to a cantilevered beam by glue. To understand the effects of the strain transmission response of the interface, pure tension loading is applied to the structure. The deformation of the mesh and the interface between the sensor and the host material when subjected to pure tension is shown in Fig. 2.

The results from the FEM simulation are shown in Table I and Fig. 3. Due to the thickness of the different interfaces and their corresponding Young's moduli, various FBG strains are obtained. The strain transmission rate is $100 \%$ when the thickness of adhesive between the FBG sensor and its host material is $2 \mu \mathrm{m}$ and the glue's Young modulus is $100 \mathrm{GPa}$. A similar result of $99 \%$ is obtained for the same glue thickness, but with a Young's modulus of $5 \mathrm{GPa}$. Little effect is found when the thickness becomes $100 \mu \mathrm{m}$ and the Young's modulus remains at $5 \mathrm{GPa}$. Note that the Young's modulus of the optical fiber is kept at $70 \mathrm{GPa}$. This clearly indicates that the thickness of the adhesive and its Young's modulus do not significantly influence the strain transmission between the optical-fiber sensor and the host material. As shown in Table I, a thinner glue with a higher Young's modulus is preferred for better strain transmission. 
TABLE I

PACKAGING EFFECTS ON TRANSMISSION RATES

\begin{tabular}{ccc}
\hline $\begin{array}{c}\text { Adhesive thickness } \\
(\mu \mathrm{m})\end{array}$ & $\begin{array}{c}\text { Young's modulus of } \\
\text { glue }(\mathrm{GPa})\end{array}$ & $\begin{array}{c}\text { Transmission rate } \\
(\%)\end{array}$ \\
\hline 2 & 100 & 100 \\
2 & 5 & 99.99 \\
100 & 100 & 98.49 \\
100 & 5 & 96.67 \\
100 (quartz-glue packaging, $0.5 \mathrm{~mm})$ & 5 & 84.36 \\
2 (steel tube packaging, $1 \mathrm{~mm})$ & 100 & 60.37 \\
2 (steel tube packaging, 2 $\mathrm{mm}$ ) & 100 & 22.07 \\
\hline
\end{tabular}

mild steel, Young's modulus $=200 \mathrm{GPa}$

optical fiber, Young's modulus $=70 \mathrm{GPa}$

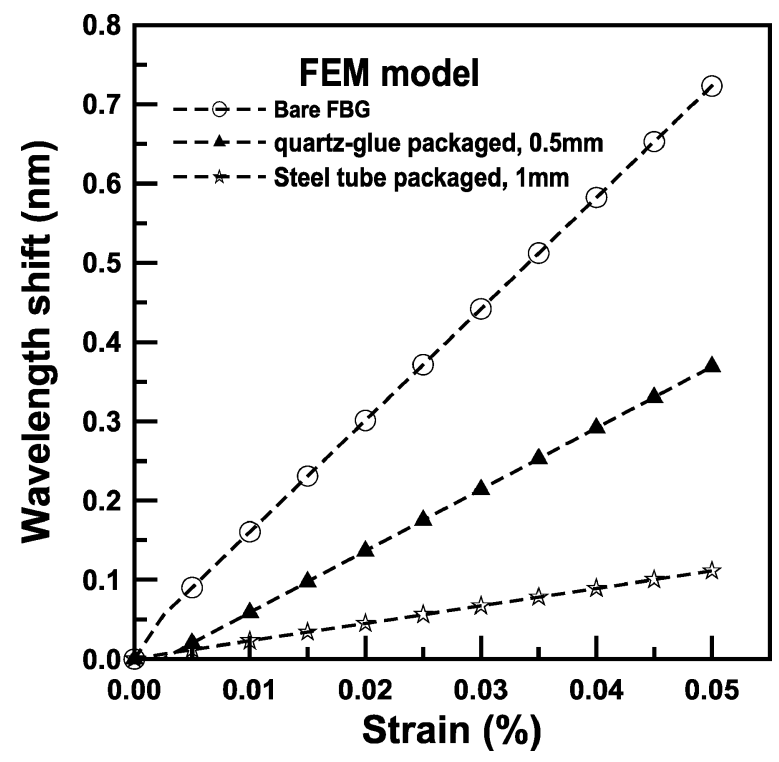

Fig. 3. Strain transmission of FEM model of the FBG sensors.

However, when the FBG sensor is recoated by quartz glue with a thickness of $0.5 \mathrm{~mm}$, not only is stress concentration induced, but the strain transmission is also decreased to a value of 84.36\%, as shown in Table I and Fig. 3. The effect of steel tube packaging is also simulated. When the wall-thickness of the steel tubes is 1 or $2 \mathrm{~mm}$, the strain transmission rates become $60.37 \%$ and $22.07 \%$, respectively. Consequently, the strain transmission rate for steel tube packaging depends heavily on the wall thickness of the steel tubing. In summary, the thickness and Young's modulus of glues have little influence on the strain transmission, especially when the thickness of the glue is less than the diameter of an optical fiber. However, recoating and steel tube packaging does significantly affect the strain transmission rate from the host material to the FBG sensor.

\section{EXPERIMENTAL SETUP AND RESULTS}

To protect the FBG sensor from being damaged, three novel packaging methods, namely, nickel recoating, quartz-glue packaging, and steel tube packaging were developed and evaluated. The packaged FBG sensors were surface attached to an ASTM-E8 specimen for measuring the Bragg wavelength shift induced by the applied loading, and the strain transmission effects were evaluated between the FBG sensor and the host

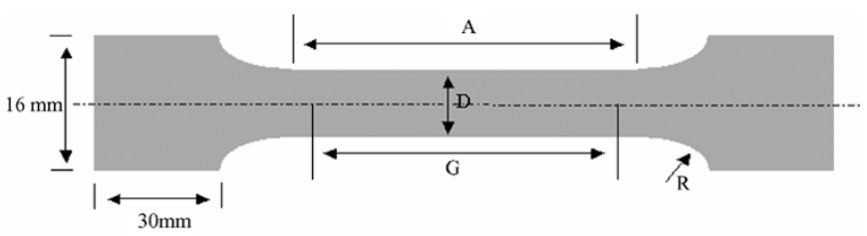

A:75 mm D: $12.5 \mathrm{~mm}$ G: $\mathbf{6 2 . 5} \mathrm{mm} \quad$ R: $10 \mathrm{~mm}$

A: length of reduced section

D: diameter

G: gauge length

$R$ : radius of fillet

Fig. 4. Schematic diagram of an ASTM-E8 specimen.

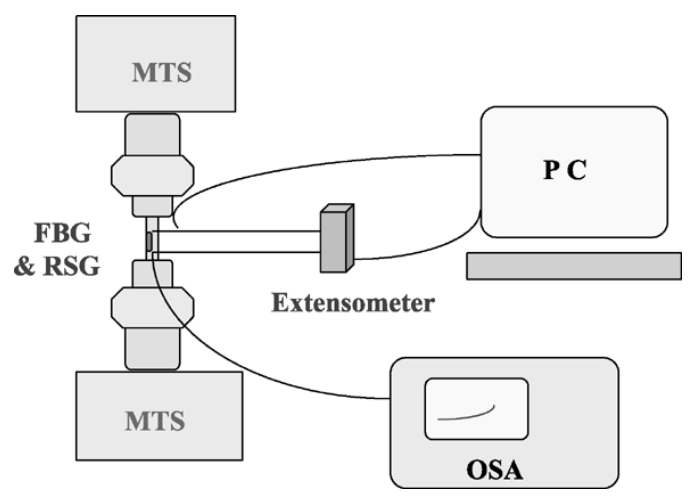

Fig. 5. Schematic diagram of the experimental setup.

material. For comparison, a conventional resistant type strain gauge (RSG, FLA-10-11, Tokyo Sokki Kenkyujo Co., Ltd.) was used in addition to the FBG sensor on the specimen, which was bonded with M-bond 200. A schematic diagram and related dimensions of the ASTM-E8 specimen are shown in Fig. 4. The ASTM-E8 specimen made of aluminum was annealed in advance to eliminate the initial strain. As shown in Fig. 5, the specimen with sensors was tested by a material test machine (MTS), which applies axial tension and compression forces at a constant temperature. The extensometer placed in close proximity to the FBG sensor was used to control the MTS loading system and to measure the strain in the specimen. An optical-spectrum analyzer (OSA, Anritsu-MS9710A) with a resolution of $0.07 \mathrm{~nm}$ was used to measure the wavelength shift of the FBG under axial deformation. An FBG-IS system (Micron Optics, Inc.) with 0.001-nm resolution and 1-s sampling rate was used for comparative measurements.

The thickness of the recoated nickel on the FBG was between 2 and $10 \mu \mathrm{m}$ by electroless plating, which has good bonding strength and uniformity. The quartz glue used to package the FBG is a material made of polymer and glass, having properties similar to an adhesive material and glass. The quartz glue was coated onto the FBG with a thickness of approximately $0.5 \mathrm{~mm}$ and was then kept at a fixed temperature for $24 \mathrm{~h}$ for solidification. Regarding the steel tube packaging, the FBG sensor was stretched and suspended inside the steel tube with a wall-thickness of 1- or 2-mm thickness, respectively, and the tube was then glued and sealed at both ends.

The measured results from the cyclic loading with ASTM-E8 specimen, the wavelength shift by the FBG sensor and the strains by a conventional sensor are shown in Fig. 6, and they 


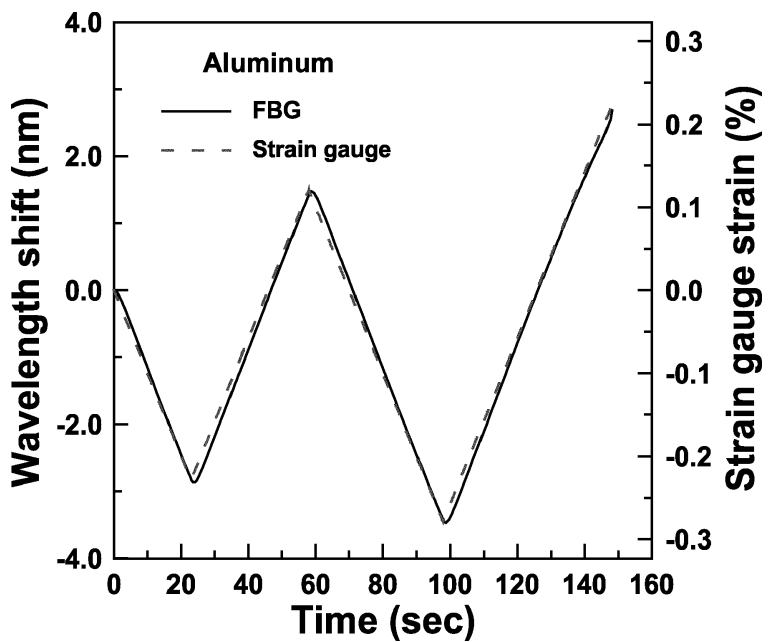

Fig. 6. Strain response of ASTM-E8 specimen from cyclic loading.

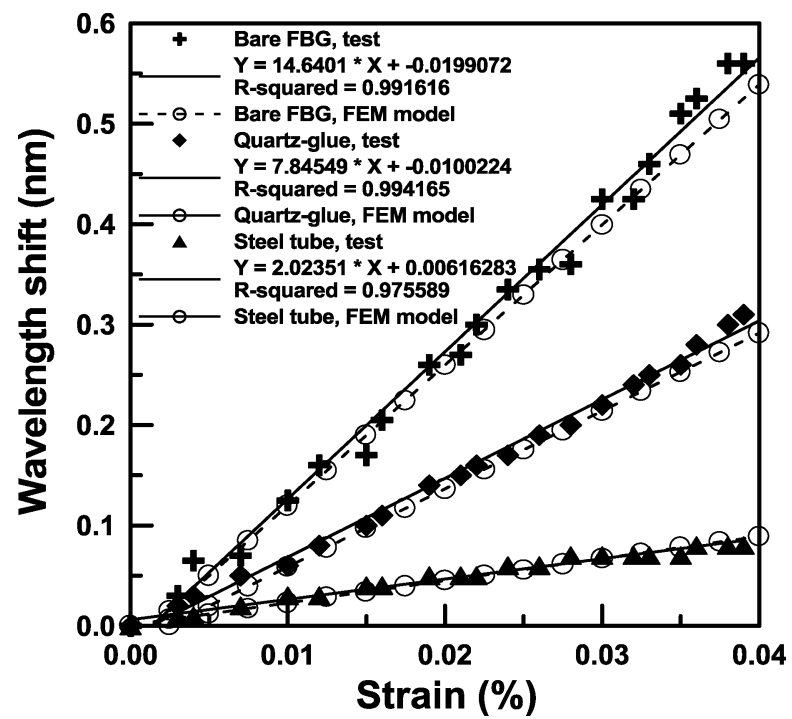

Fig. 7. Comparison of package FBG sensor with FEM model and experimental results.

indicate very good agreement between these two measurement techniques. Fig. 7 shows that good agreement is obtained between the simulated and the measured results when the FBG sensors are either quartz glue or packaged in steel tubing. Also indicated in Fig. 7 is a comparison of their sensitivities to the applied strain. Strain transmissions of $85 \%$ and $60 \%$ are obtained for the quartz-glue packaging and the steel tubing packaging, respectively. Note that the quartz-glue packaged FBG may be unreliable because of the aging problem associated with using polymer material as a glue. The steel tube packaged FBG can be used in a high-strain situation to measure large deformations due to its low sensitivity to strain transmission. As for the nickel-coated FBG sensor, which is free from the aging problem, it was surface attached to an A450 aluminum specimen and compared with a bare FBG when both were subjected to a relatively large deformation.Fig. 8 shows that the strain responses of the FBG sensor recoated with $2-\mu \mathrm{m}$-thick nickel are similar to the bare FBG under loading. Similar to the steel tube packaging results, the strain transmission decreased with the increase in thickness of the recoating nickel, as shown

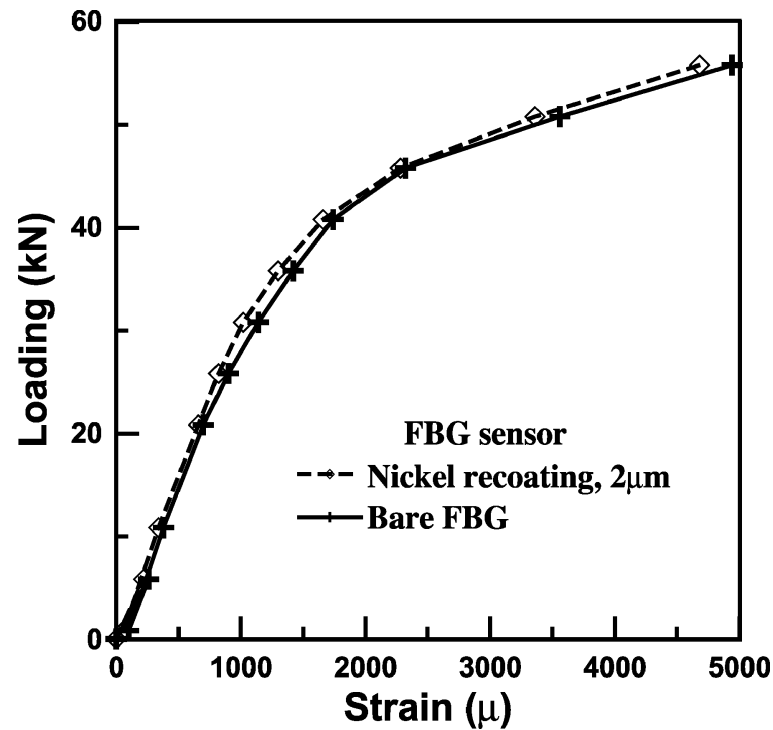

Fig. 8. Comparison of FBGs under loading with and without nickel coating.

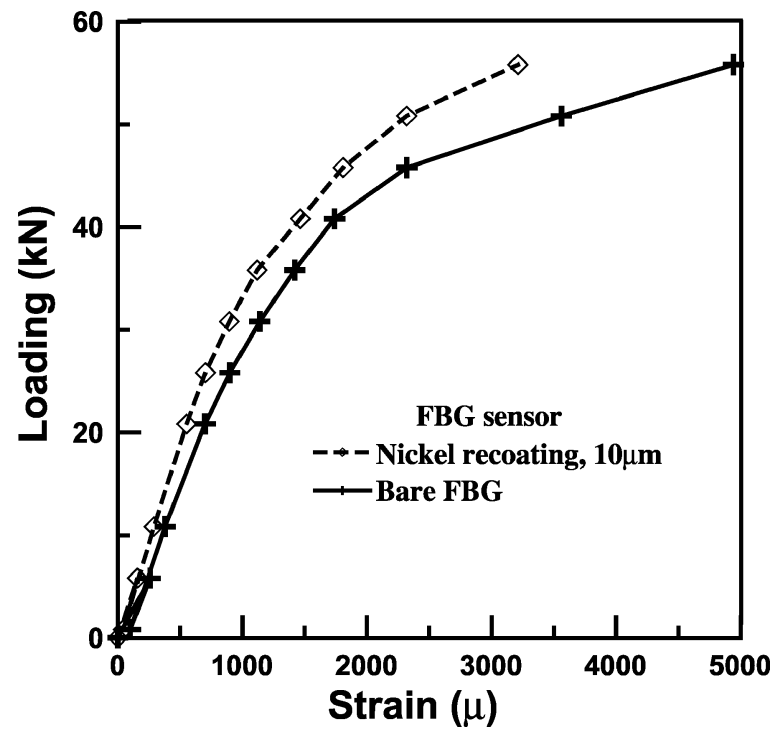

Fig. 9. Comparison of FBGs under loading with and without nickel coating.

in Fig. 9. It is shown that the nickel recoated FBG sensor can withstand larger strains than the bare FBG sensor. Fig. 10 shows the results measured by these three methods over a large strain range. It is found that the steel tube packaging method provides good protection for large deformation measurements in some civil engineering applications, since it has the least strain sensitivity. It must be pointed out that the strain transmission rates decrease with the increase in thickness of the steel tube. Based on the above simulated and experimental results, the nickel recoating method is shown to have good strain transmission efficiency when compared to other packaging methods. Furthermore, the metallic recoated membrane not only increases the strength and the durability of the FBG sensor, but also provides good compatibility for the FBG sensor to be embedded into or attached onto a civil structure. The metallic recoating method can, therefore, be expected to have a versatile potential for packaging an FBG sensor for different applications such as chemosensory receptors, medical science or environmental 


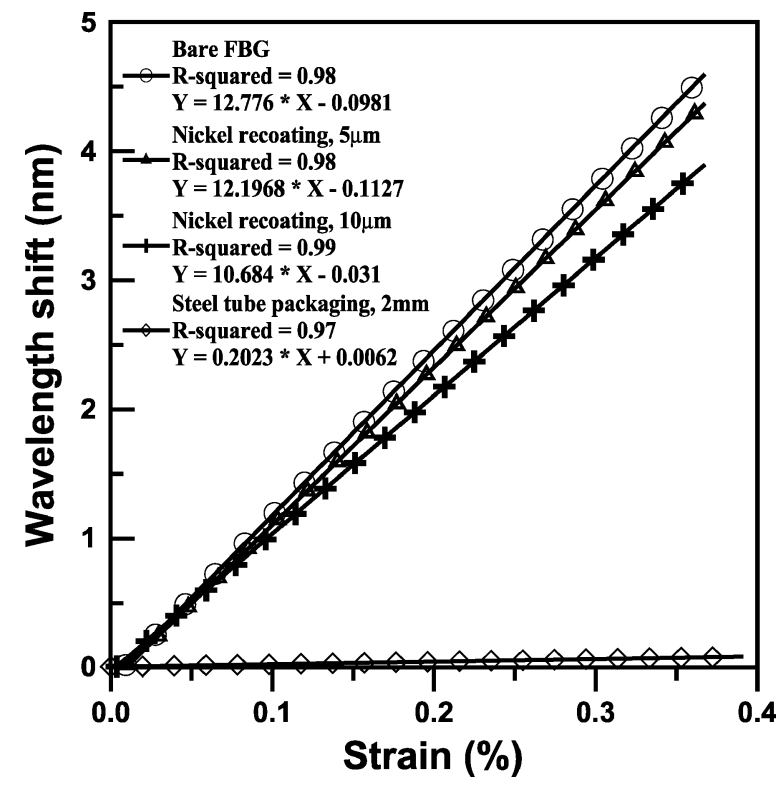

Fig. 10. Strain responses of three packaging methods.

engineering. However, the packaging processes of the metallic diffusion mechanism and the residual stress will require further research.

\section{CONCLUSION}

FBG sensors made on a bare fiber are easily damaged when handled improperly during and after fabrication. To prevent such damage, recoating the bare fiber or providing some protective packaging is generally desirable. It must be noted that glue is normally used to attach the FBG sensor onto the host material to measure strain. The aging problem of the glue must be considered since the service life of most structures is generally designed for 50 years or longer. The metallic recoated FBG sensor developed in this work used different approaches such as low-temperature solder welding for installing the sensors in the structures, a method that shows no aging problem. Also, an embedded FBG sensor must be packaged carefully to prevent corrosive damage as a result of the highly alkaline, $\mathrm{pH}-12$ concrete environment. To protect the sensor from such damage, novel packaging methods involving nickel recoating, quartz glue, and steel tubing were developed in this research. An analytical finite element model characterizing the strain transmission efficiency of the packaged FBG sensors was also developed, and the results were compared with the experimental data. It was concluded that the thickness and Young's modulus of the glues have little influence on the strain transmission, especially when the thickness of the glue is less than the diameter of an optical fiber. However, recoating and steel tube packaging will markedly affect the strain transmission rate.

It was found that the steel tube packaging method provides good protection for large deformation measurements for some civil engineering applications since it has the least strain sensitivity. It should be noted that the strain transmission rates decrease with the increase in thickness of the steel tube. Based on the above simulated and experimental results the nickel recoating method is shown to have good strain transmission efficiency in comparison with other packaging methods.

\section{REFERENCES}

[1] M. B. Kodindouma, R. L. Idriss, A. D. Kersey, M. A. Davis, D. G. Bellemore, E. J. Friebele, and M. A. Putnam, "Damage assessment of a full-scale bridge using an optical fiber monitoring system," Proc. SPIE, vol. 2719, pp. 265-275, 1996.

[2] X. Jin, J. S. Sirkis, and J. K. Chung, "Optical fiber sensor for simultaneous measurement of strain and temperature," Proc. SPIE, vol. 3042 , pp. 120-127, 1997.

[3] T. C. Kirkpatrick, D. O. Peterson, P. J. Rossi, L. R. Ray, and R. A. Livingston, "Preliminary study to facilitate smart structure systems in bridge girders," Proc. SPIE, vol. 3671, pp. 152-160, 1999.

[4] C. C. Chan, W. Jin, H. L. Ho, D. N. Wang, and Y. Wang, "Improvement of measurement accuracy of fiber Bragg grating sensor systems by use of gas absorption lines as multi-wavelength references," Electron. Lett., vol. 37, pp. 742-743, 2001.

[5] Y. Zhang, D. Feng, Z. Liu, Z. Guo, and X. Dong, "High-sensitivity pressure sensor using a shielded polymer-coated fiber Bragg grating," IEEE Photon. Technol. Lett., vol. 13, no. 4, pp. 618-619, Apr. 2001.

[6] D. F. Murphy, D. A. Flavin, and R. McBride, "Interferometric interrogation of in-fiber Bragg grating sensors without mechanical path length scanning," J. Lightw. Technol., vol. 19, no. 7, pp. 1004-1009, Jul. 2001

[7] C. C. Kuo, "The mechanical behavior research of optical fiber grating sensors," M.S. thesis, Department of Civil Engineering, National Taiwan University, Taipei, Taiwan, R.O.C., Jun. 1997.

[8] Y. J. Rao, M. R. Cooper, D. A. Jackson, C. N. Pannell, and L. Reekie, "Simultaneous measurement of displacement and temperature using in-fiber-Bragg-grating-based extrinsic Fizeau sensor," Electron. Lett., vol. 36, pp. 1610-1612, 2000.

[9] A. V. Koulaxouzidis, M. J. Holmes, C. V. Roberts, and V. A. Handerek, "A shear and vertical stress sensor for physiological measurements using fiber Bragg gratings," in Proc. IEEE 22nd Annu. Int. Conf. Engineering in Medicine and Biology Soc., vol. 1, Jul. 2000, pp. 55-58.

[10] D. K. Nath, G. W. Nelson, S. E. Griffin, C. F. Harrington, Y.-F. He, L. J. Reinhart, D. C. Paine, and T. F. Morse, "Polyimide-coated embedded optical fiber sensors," Proc. SPIE, vol. 1489, pp. 17-32, 1991.

[11] S. Yang, H. Meng, X. Dong, S. Yung, and X. Dong, "Electric current measurement with high resolution using FBG covered by aluminum thin film," Proc. SPIE, vol. 4595, pp. 200-203, 2001.

[12] K. Satori, K. Fukuchi, Y. Kurosawa, A. Hongo, and N. Takeda, "Polyimide-coated small-diameter optical fiber sensors for embedding in composite laminate structures," Proc. SPIE, vol. 4328, pp. 285-294, 2001.

[13] B. Sutapun, M. Tabib-Azar, and A. A. Kazemi, "Fiber optic Bragg grating sensors for hydrogen gas sensing," Proc. SPIE, vol. 3740, pp. 278-283, 1999.

[14] W. R. Habel, "Fiber optic sensors in civil engineering: experiences and requirements," Proc. SPIE, vol. 2509, pp. 134-141, 1995

[15] W. R. Habel and B. Hillemeier, "Results in monitoring and assessment of damages in large steel and concrete structures by means of fiber optic sensors," Proc. SPIE, vol. 2446, pp. 25-36, 1995.

[16] C. Y. Wei, S. W. James, C. C. Ye, R. P. Tatam, and P. E. Irving, "Influence of process route on mechanical and sensing performance of fiber Bragg grating optical sensors," Proc. SPIE, vol. 3670, pp. 164-170, 1999.

[17] E. R. Lyons, C. Jensen-McMullin, Y. Jiang, and H. P. Lee, "Etched cladding tunable fiber Bragg grating filters integrated with $\mathrm{Si}$ V-grooves," Proc. SPIE, vol. 4290, pp. 128-135, 2001.

[18] R. C. Foedinger, D. L. Rea, J. S. Sirkis, C. S. Baldwin, J. R. Troll, R. Grande, C. S. Davis, and T. L. Vandiver, "Embedded fiber optic sensor arrays for structural health monitoring of filament wound composite pressure vessels," Proc. SPIE, vol. 3670, pp. 289-301, 1999.

[19] W. R. Habel, M. Hoepcke, F. Basedau, and H. Polster, "Influence of concrete and alkaline solutions on different surfaces of optical fibers for sensors," Proc. SPIE, vol. 2361, pp. 168-171, 1994.

[20] H.-H. Tsai, W.-Y. Jang, and F.-F. Yeh, "Central wavelength tunable mechanism for temperature compensated package of fiber Bragg gratings," IEEE Trans. Adv. Packag., vol. 24, no. 1, pp. 86-90, Feb. 2001.

[21] Y.-L. Lo and C.-P. Kuo, "Packaging a fiber Bragg grating without preloading in a simple athermal bimaterial device," IEEE Trans. Adv. Packag., vol. 25, no. 1, pp. 50-53, Feb. 2002.

[22] Computers and Structures, Inc., Berkeley, CA, SAP 2000 Integrated Finite Element Analysis and Design of Structures, Sep. 1997. 


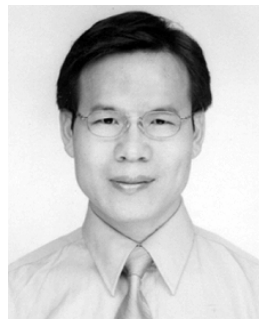

Yung Bin Lin received the Ph.D. degree in civil engineering from the National Taiwan University, Taipei, Taiwan, R.O.C., in 2000.

His educational background focused on sensors development and its applications. Before joining the National Center for Research on Earthquake Engineering (NCREE), Taipei, he was a Visiting Assistant Professor for one year at the Department of Photonics, National Chaio-Tung University, Hsinchu, Taiwan, and conducted three years of research at Materials Research Laboratories, Industrial Technology Research Institute, Hsinchu. He has worked on the development of optical-fiber sensors and monitoring system development.

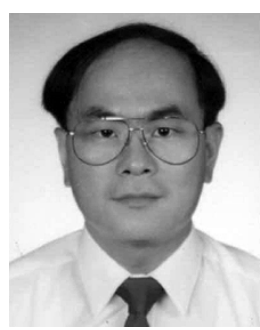

Kuo Chun Chang received the Ph.D. degree in civil engineering from the State University of New York at Buffalo in 1985.

His education background focused on inelastic behavior of structures. Before joining National Taiwan University, Taipei, Taiwan, R.O.C., he was a Research Associate Professor from 1985 to 1991 at the State University of New York at Buffalo, carrying out structural control research. His current research interests include seismic retrofit of structures, structural control, and health monitoring.

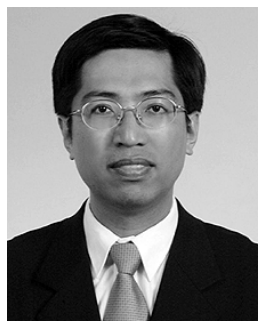

Jenn Chuan Chern is a Professor of civil engineering at the National Taiwan University, Taipei, Taiwan, R.O.C. His research interests include high-performance concrete, bridge engineering, and advanced materials.

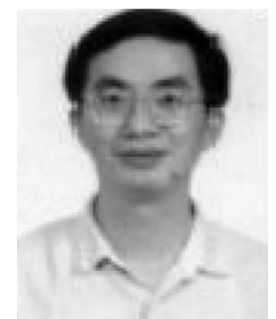

Lon A. Wang received the Ph.D. degree from the Optical Sciences Center, University of Arizona, Tucson, in 1988.

After receiving the Ph.D. degree, he continued as a Postdoctoral Researcher. In 1989, he joined Bell Communication Research, where he worked in the areas of wavelength division multiplexing technologies and optical-fiber network system technologies. In 1992, he joined the Institute of Electro-Optical Engineering and the Department of Electrical Engineering, National Taiwan University, Taipei, Taiwan, R.O.C., where he is currently a Professor. His current interests are in the design, fabrication, and modeling of active and passive fiber devices and guided-wave components for photonic integrated circuits, optical-fiber communication, and sensing-system applications, as well as semiconductor nanofabrication for integrated circuits and electrooptical devices. 